\section{ORIGINAL RESEARCH}

O. Majdani

K. Thews

S. Bartling

M. Leinung

C. Dalchow

R. Labadie

T. Lenarz

G. Heidrich

\title{
Temporal Bone Imaging: Comparison of Flat Panel Volume CT and Multisection CT
}

BACKGROUND AND PURPOSE: A recent development in radiology is the use of flat panel detectors in CT to obtain higher-resolution images. This technique is known as flat panel volume CT (fpVCT). We sought to compare the image quality and diagnostic value of 2 different flat panel detector-equipped scanners: one is a prototype fpVCT scanner, and the other is a so-called flat panel digital volume tomography (fpDVT) scanner, which is routinely used in clinical setup with current state-of-the-art multisection CT (MSCT) scanners.

MATERIALS AND METHODS: Five explanted temporal bones and 2 whole-head cadaveric specimens were scanned with fpVCT, fpDVT, and MSCT scanners. The image series were blindly evaluated by 3 trained observers who rated 38 anatomic structures with regard to their delineation/appearance.

RESULTS: Although the image quality obtained with fpVCT and fpDVT was rated significantly better compared with MSCT on isolated temporal bones, the differences were not significant when whole cadaveric heads were scanned.

CoNCLUSIONS: Theoretic and practical advantages exist for flat panel detector-equipped scanners, including improved image quality. However, when imaging whole cadaveric heads, no significant difference could be demonstrated between them and standard-of-care MSCT.

D iagnostic imaging of the lateral skull base requires high resolution because many anatomic structures of interest are diminutive, including the ossicles (which have dimensions ranging from $40 \mu \mathrm{m}$ to $4 \mathrm{~mm}$ ) and the cochlear aqueduct (which has an average diameter of $197 \mu \mathrm{m}$ ). Despite their small size, pathologic changes to these structures can have a large impact on health, including diminished hearing and balance. Current radiographic imaging of these structures approaches the resolution of multisection CT (MSCT) scanners, often necessitating more invasive diagnostic modalities such as surgical exploration. Improved resolution of CT scanners could potentially eliminate the need for exploratory surgery of this nature.

For the past 2 decades, imaging of the lateral skull base has evolved from single, flat-plate radiographs involving creative positioning of the patient (eg, Schüller and Stenver projection) to the development of the multisection spiral CT technique, which minimizes acquisition time and allows images relatively free of motion artifacts to be obtained. ${ }^{1,2}$ The latest development in this field has been the use of flat panel detectors that allow improved spatial resolution. Flat panel detectors consist of isometric sensors (CsI crystals), which are ordered in an array placed on photodiode transistors. These transistors absorb light produced when the CsI crystals absorb $\mathrm{X}$-rays and convert these into electrical impulses, which can be displayed as a radiographic image. Despite the theoretic im-

Received November 10, 2008; accepted January 29, 2009.

From the Department of Otolaryngology (O.M., K.T., M.L., T.L.), Hannover Medical School, Hannover, Germany; Department of Radiology (S.B.), German Cancer Research Centre, Heidelberg, Germany; Department of Otolaryngology (C.D.), Park Klinik Weissensee, Berlin, Germany; Department of Otolaryngology (R.L.), Vanderbilt University Medical Center, Nashville, Tenn; and Department of Radiology (G.H.), Georg-August University of Göttingen, Germany.

Please address correspondence to Omid Majdani, MD, PhD, Hannover Medical School, Otolaryngology Head and Neck Surgery, Carl-Neuberg-Str 1, OE 6500, 30625 Hannover, Germany; e-mail: majdani.omid@mh-hannover.de

Eindicates article with supplemental on-line table.

DOI 10.3174/ajnr.A1560 provement in spatial resolution that flat panel volume CT (fpVCT) allows, other parameters such as soft-tissue attenuation and susceptibility to radiation scatter may limit its clinical usefulness.

We sought to investigate the potential advantage of fpVCT systems in the imaging of temporal bone anatomy. We report herein on the delineation of small anatomic structures within the temporal bone subsequent to both fpVCT and MSCT. Initial studies were conducted with isolated temporal bones according to availability. However, knowing that larger tissue masses pose additional challenge to CT scanner systems, we also included cadaveric "whole head" specimens.

\section{Materials and Methods}

Three CT scanners were used to scan 5 cadaveric temporal bones and 2 cadaveric heads. To mimic postoperative conditions, the temporal bones were dissected before scanning. To investigate scanning involving maximal tissue volume, the whole-head preparations were not dissected before scanning.

The 3 CT scanners used were: a MSCT scanner (VFX16 scanner; GE Healthcare, Milwaukee, Wis), a flat panel digital volume tomography scanner (fpDVT) (Accu-I-Tomo; J. Morita Manufacturing, Kyoto, Japan), and a fpVCT prototype scanner (GE Healtcare). To ensure meaningful comparison between these scanners, modular transfer function (MTF) is reported for each scanning protocol. The MTF is used to report resolution of objects.

\section{Imaging}

We scanned each specimen by using a 16-section scanner, a fpDVT scanner, and a fpVCT scanner. Spatial resolution is defined as the smallest separation at which 2 objects can be distinguished as 2 separate entities. A parameter for comparing the quality of the spatial resolution of the different scanners is MTF or the line pairs per centimeter $(\mathrm{lp} / \mathrm{cm})$. The higher the spatial frequency at $10 \%$ modulation transfer, the higher the spatial resolution is and, hence, the finer the details in the CT image. ${ }^{3}$ 


\section{Multisection CT}

The Lightspeed VFX16 scanner (GE Healthcare) is a 16-slice CT scanner used in routine clinical practice. The minimum section thickness of this device is $0.625 \mathrm{~mm}$. It is fitted with a HiLight Matrix 2 detector, consisting of 21.888 detector elements. The voxel dimensions (voxel $=$ volume pixels $)$ of the resulting image were $0.1875 \mathrm{~mm}(\mathrm{x}$ direction $) \times 0.1875 \mathrm{~mm}(\mathrm{y}) \times 0.625 \mathrm{~mm}(\mathrm{z})$ : the voxels were anisotropic. The scanner is manufactured with an MTF of $13.9 \mathrm{lp} / \mathrm{cm}$ at $10 \%$ MTF.

The scan parameters for the temporal bones were as follows: current, $30 \mathrm{~mA}$; voltage, $120 \mathrm{kV}$; pitch, 0.562:1; rotation time, 1 second; section collimation, $0.625 \mathrm{~mm}$; matrix, $512 \times 512$; FOV, $9.6 \mathrm{~cm}$; and reconstruction increment, $0.2 \mathrm{~mm}$.

For the whole head, the same parameters were applied (except for the current, which was $120 \mathrm{~mA}$ ). Use of this protocol involves a radiation CT dose index of approximately $32.3 \mathrm{mGy}$.

\section{Flat Panel-Based Digital Volume Tomography}

The Accu-I-Tomo (J. Morita Manufacturing) is a digital volume tomography scanner that was developed for imaging of the head region and is often used for dental imaging. The minimum section thickness of this device is $0.125 \mathrm{~mm}$. The MTF is approximately $20 \mathrm{lp} / \mathrm{cm}$.

The scan parameters for the temporal bones were as follows: current, $8 \mathrm{~mA}$; voltage, $60 \mathrm{kV}$; rotation time, $17.5 \mathrm{~s}$; section collimation, $0.125 \mathrm{~mm}$; matrix, $481 \times 481$; FOV, $6 \mathrm{~cm} \times 6 \mathrm{~cm}$; and reconstruction increment, $0.125 \mathrm{~mm}$. The radiation dose in this setup was $0.12 \mathrm{mGy}$. To increase photon penetration, the voltage was increased to $80 \mathrm{kV}$ for the whole head, with all other parameters left unchanged.

The voxel dimensions of the resulting dataset were $(0.125 \mathrm{~mm})^{3}$; the voxels were isotropic. The data were saved as $3 \mathrm{D}$ volume data and was converted to DICOM 3D with use of the device's application software (i-Dixel). Several image processors are available; the highresolution bone algorithm filter was applied to the examined image data.

\section{Flat Panel-Based Volume CT}

The fpVCT scanner used for our study is a prototype device manufactured by GE Healthcare. This device has not yet been licensed for human application. The minimum section thickness of this device is $0.145 \mathrm{~mm}$. The resolution is approximately $36 \mathrm{lp} / \mathrm{cm}$.

The scan parameters used for the temporal bones were as follows: current, $40 \mathrm{~mA}$; voltage, $120 \mathrm{kV}$; rotation time, $8 \mathrm{~s}$; section collimation, 0.145 to $0.190 \mathrm{~mm}$; reconstruction matrix, $1024 \times 1024$ or alternatively $512 \times 512$. Each flat panel detector element has a size of $0.2 \times 0.2 \mathrm{~mm}^{2}$. The acquired image data voxels are isotropic. The FOV is $12.8 \times 12.8 \times 4.2 \mathrm{~cm}^{3}(\mathrm{x}, \mathrm{y}, \mathrm{z})$ when using the 1 -detector mode. With use of both built-in detectors, the FOV can be increased to $33.5 \times 33.5 \times 3.5 \mathrm{~cm}^{3}(\mathrm{x}, \mathrm{y}, \mathrm{z})$. The acquisition length can be enhanced to $21 \mathrm{~cm}$ in the $\mathrm{z}$-direction by means of a step-and-shoot acquisition technique. The effective detector area is $33.5 \times 33.5 \mathrm{~cm} \times$ $21 \mathrm{~cm}$, sufficient for a scan of a whole human head.

For the whole head, the same parameters were applied (apart from the section thickness, which was $0.2-0.26 \mathrm{~mm}$ ). The voxel (volume pixels) dimensions of the resulting imaging were approximately $(0.15$ $\mathrm{mm})^{3}$; the voxels were isotropic. The image data were saved as $3 \mathrm{D}$ raw data and were converted to DICOM3 format in $0.15-\mathrm{mm}$ sections. Because this machine is not considered for use in human subjects, we did not measure the radiation dose.

\section{Investigators}

Each scan was evaluated by 3 examiners. Two were board certified neuroradiologists, and the third was a senior and board certified physician specializing in neuroanatomy and experienced in middle-ear anatomy.

\section{Measurement Method}

Volume Viewer 2 software (Voxtools; GE Healthcare, Milwaukee, Wis) on an Advantage Workstation 4.1 (GE Healthcare) was used for visually reproducing the data. The investigator was permitted to scroll through the image sections and perform reconstructions of the volume data, and to zoom in and out in any order. In its initial mode, the Volume Viewer 2 software displays 4 windows on the screen corresponding to the axial, coronal, sagittal, and oblique sections of the $3 \mathrm{D}$ volume. The DICOM data were anonymized beforehand to ensure that the examiner did not know which kind of scanner was used for the imaging.

Each anatomic structure was rated on a scale of 0 to 3 , in which 0 indicated the anatomic structure could not be identified; 1 , the anatomic structure could not be easily delineated from the surrounding structures; 2 , the structure was well delineated from the surrounding structures; and 3, the structure was very well delineated from the surrounding structures. The results for identification of the different anatomic structures were calculated in percentage rates on the basis of this rating scale. Thirty-eight structures were evaluated for each image dataset (on-line Table).

\section{Statistical Analysis}

We performed statistical analysis using SPSS 13.0 software (SPSS, Chicago, Ill). We used the Mann-Whitney $U$ test, which is a nonparametric test to assess whether 2 samples of observations belong to the same distribution, to analyze whether there is a difference between the imaging of isolated temporal bone specimens and that of whole-head specimens.

We used the Kruskal-Wallis test, which is a nonparametric method to test the equality of population medians among groups. It is an extension of the Mann-Whitney $U$ test to 3 or more groups; the test assumes an identically-shaped distribution for each group, except for any difference in medians. We used the Kruskal-Wallis test to compare the different imaging modalities (fpVCT, fpDVT, and MSCT) to image the isolated temporal bone specimen and for the whole-head specimens.

\section{Results}

The results are detailed in the accompanying Table. The tympanic membrane could be best identified in the isolated temporal bones by flat panel devices. With whole-head specimens, the identification of the tympanic membrane was only possible in $47 \%$ to $61 \%$ of cases with either scanner. In isolated temporal bone specimens, the ossicular chain substructures are best identified by fpVCT $(85.7 \% \pm 14.4 \%)$; with the fpDVT scanner, the structures can be identified in $76.0 \% \pm$ $17.0 \%$ of cases. Both methods are superior to the MSCT method with its $59.2 \% \pm 18.5 \%$ detail resolution for the ossicles and their substructures (on-line Table and Figs 1-3).

The difference between the 3 different scanners with regard to imaging of the temporal bone is much less for the wholehead structures (Fig 3). In the whole-head specimen, the substructures of the ossicular chain are best distinguished from adjacent structures when imaging is performed with fpDVT 
$(68.5 \% \pm 16.7 \%)$. The quality of imaging was approximately that of MSCT imaging $(63.4 \% \pm 19.1 \%)$ and fpVCT imaging $(66.0 \% \pm 19.6 \%)$.

In explanted temporal bone specimens, stapes substructures such as the caput, collum, basis of the stapes, and stapedius muscle were distinguishable from the surrounding structures in $33.3 \%$ to $57.8 \%(45.9 \% \pm 11.1 \%)$ of cases in MSCT imaging, in $55.6 \%$ to $73.3 \%(63.7 \% \pm 8.5 \%)$ of cases in fpDVT imaging, and in $66.7 \%$ to $93.3 \%(79.6 \% \pm 10.4 \%)$ of cases in fpVCT imaging (Fig 1). The cochlear and vestibular aqueducts were clearly identified in $44.4 \%$ and $51.1 \%(47.8 \% \pm 4.7 \%)$ of the images created with an MSCT scanner, in $75.6 \%$ and $82.2 \%(78.9 \% \pm 4.7 \%)$ when imaging was performed with the fpDVT scanner, and $75.6 \%$ for both structures when fpVCT imaging was performed (Fig 2).

In whole-head specimens, each substructure of the stapes proved clearly visible in approximately $38.9 \%$ to $69.4 \%$ $(51.9 \% \pm 12.7 \%)$ with use of the MSCT scanner, whereas fpDVT revealed these structures in $44.4 \%$ to $63.9 \%$ (56.02\% 土 $9.5 \%$ ), and fpVCT imaging showed them in $30.6 \%$ to $66.7 \%$ $(50.9 \% \pm 12.9 \%)$ of cases. The cochlear and vestibular aqueducts were clearly visible-specifically, in $52.8 \%$ and $72.2 \%$ of cases with MSCT, $69.4 \%$ and $75 \%$ with fpDVT, and $69.4 \%$ and $72.2 \%$ with fpVCT (Fig 2).

For imaging of the cochlear substructures in whole-head specimens, the lamina spiralis ossea cochleae and the round window were best visualized with use of the fpVCT scanner (47.2\% and 75\%), whereas the bony labyrinth was most clearly imaged (100\%) with MSCT and fpDVT. With use of fpVCT, the examiners gave the bony labyrinth an overall rating of $88.9 \%$. The modiolus cochleae were best visualized by MSCT (88.9\%; Fig 2).

The substructures of the inner ear canal (the main, superior, and inferior branches of the vestibular nerve canal, as well as the cochlear nerve canal) were best visualized by the fpVCT scanner, and the second-best option proved to be fpDVT with whole-head specimens.

In comparison of the different examiners' output results for the temporal bone and the whole-head specimens, without making allowances for the different scan modes, the results were significantly different (Mann Whitney- $U$ test: $P<.001$ ).

The Kruskal-Wallis test was applied to differentiate the rank sum for the 3 different scanners without differentiating between whether the temporal bone specimens or the wholehead specimens were evaluated. The difference among all scanners was highly significant $(P<.001)$. The fpVCT had the highest rank sum, fpDVT was in the middle range, and MSCT had the lowest rank sum. The Mann-Whitney test was then applied to differentiate between the temporal bone and the whole-head specimens. The middle rank sum for fpVCT was higher than that for fpDVT, and the middle rank sum of the fpDVT was even higher than for MSCT, but the only significant difference was for the temporal bone specimens $(P<$ $.001)$. The difference between scan modes for the whole-head specimens was not significant $(P=.086)$.

To investigate whether the rating of the 3 different examiners was homogeneous, we applied the Kruskal-Wallis test. There was a highly significant difference between the first rater and the other 2 examiners in their evaluations for the temporal bones, though no significant difference could be found
(Mann-Whitney test, $P<.001$ ) between the second and third examiners. With regard to the whole-head specimens, there was a significant difference $(P=.015)$ between the first and second examiners. The difference between the first and third examiners was highly significant $(P<.001)$, as was that between the second and third examiners.

\section{Discussion}

Refinements in CT technology have also led to advances in the $\mathrm{x}$-ray detectors used. Flat panel detectors use an array of detectors with an isovolumetric size of $200 \mu \mathrm{m}$ (edge length), whereas with single-section or MSCT scanners, a linear x-ray beam is emitted and focused so that it precisely strikes 1 dedicated $\mathrm{x}$-ray detector on the opposite side. The cone beam scanners that use flat panel detectors emit a single cone beamshaped ray that impinges on the flat panel detector on the opposite side after it having passed through the body. These scanners are therefore called volume CT scanners. ${ }^{2,4-6}$ Both conventionally equipped DVT devices and those equipped with flat panels, which are often used in dental imaging, mostly use cone beam 3D scanners. ${ }^{7-11}$ A small number of prototype CT machines have also been developed, though these have not yet been approved for clinical use.

Single-section high-resolution CT (HR-CT) provides 1-mm sections so that the submillimetric structures cannot be captured with use of this method. To improve the resolution of the HR-CT, the technique of overlapping 1-mm sections with the volume data-thus acquiring MSCT — was developed. ${ }^{1,2}$ Although details with an edge length of $0.3 \mathrm{~mm}$ can be captured with use of this method, the patient is exposed to higher radiation levels than with the regular HR-CT. Flat panel detector-based scanners have detail resolution of up to $0.125 \mathrm{~mm}^{12,13}$

We designed this study involving human cadaveric temporal bone and whole-head specimens to evaluate the diagnostic value of the new CT and DVT scanner equipped with flat panel detectors to image lateral skull base structures. This study is the first of which we are aware that compares different flat panel detector-based scanners with state-of-the-art MSCT scanners by examining isolated temporal bones and wholehead specimens.

The most important finding of this study is that there is no significant difference among the 3 different scanner types when whole heads are scanned, which implies that the flat panel-based devices have almost the same diagnostic value for scanning the lateral skull base as the CT scanner. Identification of the anatomic structures with flat panel devices is significantly better than MSCT scanning when isolated temporal bones are scanned. Although this fact is not applicable to clinical routine, it is a good method to image temporal bones for research purposes. ${ }^{14,15}$

Multisection CT scanners are state-of-the-art for imaging and are in widespread use. Patients have much greater access to these scanners than to the new-generation flat panel devices. Another advantage of multisection CT scanners is their short period of data acquisition compared with flat panel scanners, making them less susceptible to artifacts caused by patients' movement. A MSCT scanner has a rotation time of $0.375 \mathrm{~ms}$ and takes 1 to $2 \mathrm{~s}$ to image the temporal bone area. The exposure time for the fpDVT (Accu-i-tomo FPD) used 


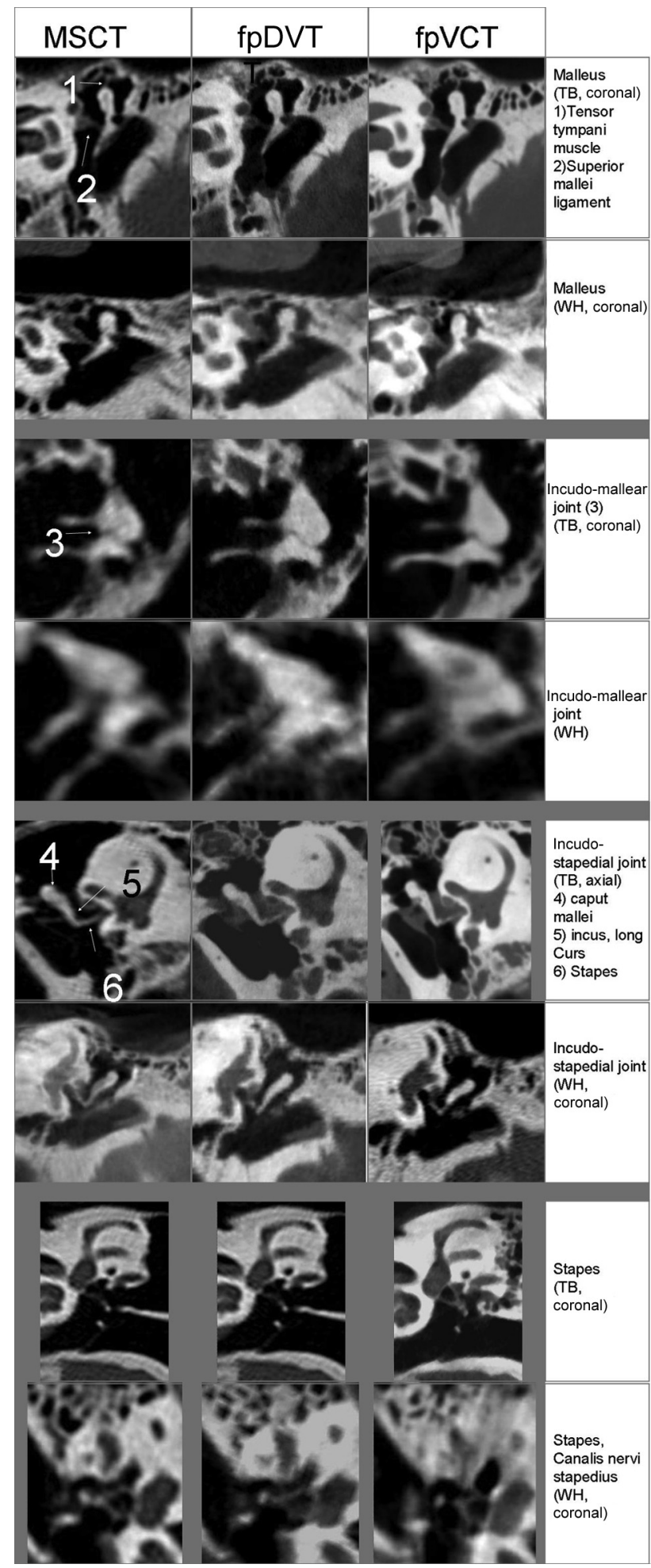

Fig 1. Images of the incus, ambomalleolar joint, incostapedial joint, and the stapes in explanted temporal bone (TB) and whole-head specimens (WH) obtained by 3 different scanners.

here is $18 \mathrm{~s}$. The fpVCT requires $8 \mathrm{~s}$ for each image sequence (ie, for each rotation); 1 image sequence is sufficient to image the temporal bone region.

Flat panel detectors in the current setting are optimized for bone and hard-tissue imaging; they have very good contrast for dental applications or for anatomic areas consisting of

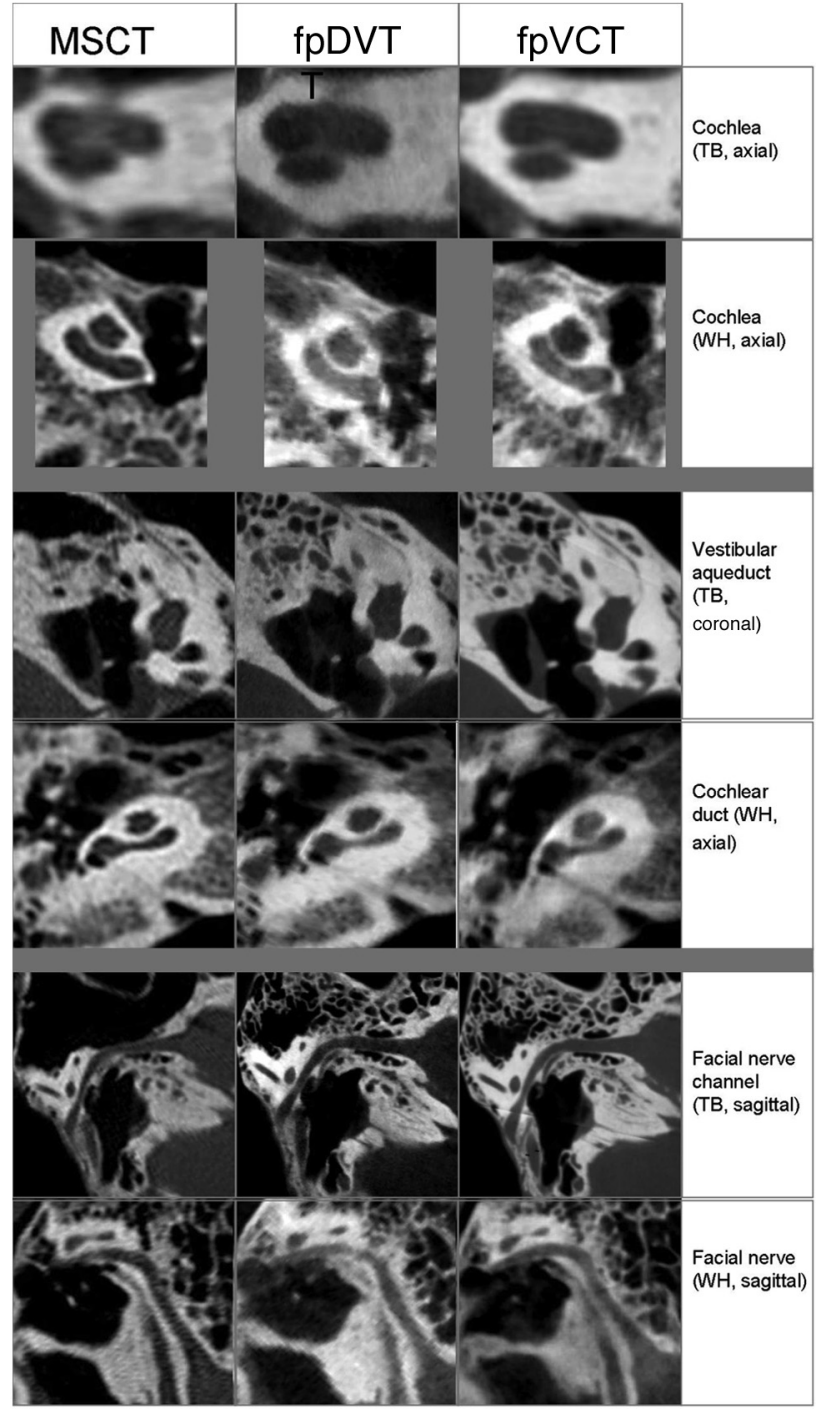

Fig 2. Images of the cochlea, vestibular duct, cochlear duct, and facial nerve in explanted temporal bone (TB) and whole-head specimens (WH).

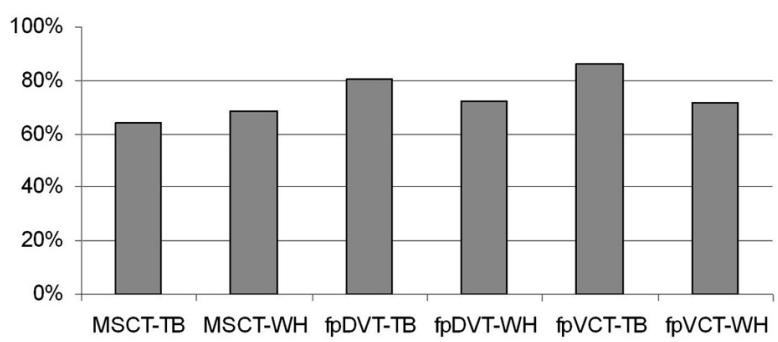

Fig 3. Three different examiners rated image quality with regard to delimitation of 38 different anatomic substructures as viewed in explanted temporal bones (TB) and wholehead (WH) specimens, each specimen being scanned with an MSCT scanner, an fpDVT scanner, and an fpVCT scanner.

bony tissue, such as the temporal bone. Soft-tissue contrast is, however, not yet sufficient for clinical diagnostic applications; therefore, these detectors cannot yet replace the state-of-theart MSCT scanner. ${ }^{1,2}$

First-generation DVT devices used the analog technique of converting the $\mathrm{x}$-ray beam for image acquisition. ${ }^{12,13}$ This included focusing of the $\mathrm{x}$-ray before it impinges on the chargecoupled device camera and analog-digital (A/D) converters. 
An A/D conversion is susceptible to artifacts as well as to electromagnetic field interferences. Another problem was that the FOV was limited to $3 \times 4 \mathrm{~cm}^{2,3}$ The new-generation flat panel detectors are less susceptible to artifacts, and the FOV extends from $6 \mathrm{~cm} \times 6 \mathrm{~cm}$ (3D-Accuitomo; J. Morita) up to $12 \mathrm{~cm} \times$ $16.5 \mathrm{~cm}$ (MiniCAT; Xoran, Ann Arbor, Mich).

The main advantage of flat panel detectors is their enhanced physical resolution, which leads to more detailed image acquisition. Another advantage of the flat panel detectors is their isotropic voxel shape, which allows performing free $3 \mathrm{D}$ reconstructions with the same resolution as in primary acquired series. The GE fpVCT flat panel detector uses arrays with $1024 \times 1024$ detector subunits; each has a size of $0.2 \times$ $0.2 \times 0.2 \mathrm{~mm}^{3}$. The acquired image data voxels are isotropic. Detail resolution in high-contrast regions is approximately $150 \mu \mathrm{m}$. The detector in the Morita Accu-i-Tomo FPD device uses similar technology. The flat panel array consists of subunits, each $(0.125 \mathrm{~mm})^{3}$ in volume. The acquired image data voxels are isotropic. The best detail resolution of this device is $125 \mu \mathrm{m}$. The 16-section CT scanner used for this study can create images with a resolution of $0.5 \times 0.5 \times 0.6 \mathrm{~mm}^{3}$.

The radiation dose to which the patients are exposed is a major issue, especially in skull base imaging, owing to the vulnerable eye lenses' exposure to radiation. Referring to the literature, the patient's radiation exposure can be lower with flat panel-based scanners than with MSCT scanners, ${ }^{8-10,12,13,16}$ which is mostly because of the reduced dynamic range of flat panel detector systems, limiting the maximal photon flux. However, image quality measurements such as signal-to-noise ratio and resolution should be considered when dose measurements of scanner setups are compared. The radiation doses mentioned in this study are provided by the manufacturer; we did not measure radiation exposure because these data are well known.,

For imaging of isolated temporal bone specimens, the detail resolution of the bony structures was best with fpVCT, but the flat panel scanners did have a similar detail resolution to MSCT when whole-head specimens were scanned. Similar findings were reported when a series of images of the axial skeleton and limbs were created with an ISO-C-3D fluoroscopy device. ${ }^{17,18}$

A comparison between fpVCT or fpDVT scans of isolated temporal bone specimens and the scans of whole-head specimens revealed that the detailed resolution of the images was higher; however, with flat panel scanners, the filigree structures of temporal bone were displayed more clearly. The images of the whole-head specimen on flat panel devices were noisier. The reasons for loss of detail resolution when imaging whole-head specimens (as opposed to isolated temporal bone specimens when scanning with flat panel detector scanners) are multifactorial: beam hardening, scattering, $\mathrm{x}$-ray permeation, and radiation absorption.

When the whole head instead of the explanted temporal bone specimen is scanned, the mass of tissue between the radiation source and the detector is greater even if the FOV does not change. Because this tissue is a kind of radiation filter itself, more low-energy radiation gets absorbed while scanning the whole-head specimen and more high-energy radiation passes through (this being termed beam hardening). ${ }^{4}$ For the same reason, the absorption of radiation of all energy levels is higher, and radiation penetration is lower with whole-head specimens.

An additional problem is the inhomogeneity of the substructures of the whole-head specimen, as revealed by different levels of radiation absorption and penetration as well as different values for beam hardening while the radiation source and detector are turning around the head. For example, the amount of bone allowed by the beam is much greater in the left-to-right direction because the 2 temporal bones overlap than for the anteroposterior view. The aim of CT imaging, namely to determine a material-specific value for each point in the target object, is hindered. Bone algorithms can be used to correct these artifacts; thus, imaging is also dependent on the use of different correction algorithms applied by different manufacturers. ${ }^{2}$

Radiation scattering causes more artifacts in examined flat panel devices than in MSCT. Scattering occurs once the emitted beam penetrates an object, and the direction of parts of the beam changes, owing to the different shape and material of the objects' substructures. The CT scanner detector is positioned directly parallel to the $\mathrm{x}$-ray source of each CT scanner; in this way, each detector cell is matched to a predefined $\mathrm{x}$-ray portion. Scattered beams may strike the wrong detector cell, which is responsible for detecting the quantum of another line integral. The detection of the primary beam thus becomes overloaded with the scattered beams. The result is loss of contrast and detail resolution in the final image. Although MSCT devices use scattering filters, these were not included in the fpVCT scanners we investigated. This is one of the reasons why flat panel scanners lose the advantage they have in detailed resolution when the explanted temporal bone specimen is imaged, compared with whole-head scanning with the MSCT device. Moreover, the scattered beams have more influence on the flat panel detectors than in MSCT devices because of the smaller voxel size of the detectors.

Unexpectedly, the quality of imaging achieved with the MSCT device was, with regard to clarity, contrast, and detail resolution, much better when imaging whole-head specimens than explanted temporal bones. This may have been the result of the optimized configuration of the MSCT detectors and the architecture of the scanner for imaging of whole-head specimens.

Another advantage of the MSCT detectors vs flat panel detectors is their higher quantum efficiency and higher dynamic range. Quantum efficiency describes the ratio of the processes run by the detector to the number of the absorbed quantum (photon). The dynamic range of a detector describes the range of radiation that it can detect.

\section{Conclusions}

We compared the image quality obtained with isolated temporal bones and temporal bone structures in human wholehead specimens while acquiring 3D image datasets by using the current state-of-the-art MSCT, fpDVT, and fpVCT scanners.

Although many published studies highlight the promising results obtained with isolated temporal bone specimens, they showed that the detail resolution of the flat panel-based devices is superior to that with MSCT. We were able to verify this finding for the isolated temporal bones and also established 
that image quality is not significantly different from that of the MSCT images in the identification of temporal bone hard tissue substructures when scanning whole-head specimens. One aspect in favor of flat panel-based devices is that patients' exposure to radiation is less than with MSCT scans; however, the quality of soft tissue imaging is a major drawback.

\section{References}

1. Kalender WA. Der Einsatz von Flachbilddetektoren für die CT-Bildgebung [The use of flat panel detectors for CT imaging]. Radiologe 2003;43:379-87

2. Kalender WA, Kyriakou Y. Flat-detector computed tomography (FD-CT). Eur Radiol 2007;17:2767-79

3. Gupta R, Grasruck M, Suess C, et al. Ultra-high resolution flat panel volume CT: fundamental principles, design architecture, and system characterization. Eur Radiol 2006;16:1191-205

4. Peltonen LI, Aarnisalo AA, Kortesniemi MK, et al. Limited cone-beam computed tomography imaging of the middle ear: a comparison with multislice helical computed tomography. Acta Radiol 2007;48:207-12

5. Ross W, Cody DD, Hazle JD. Design and performance characteristics of a digital flat panel computed tomography system. Med Phys 2006;33:1888-901

6. Webb $S$. Historical experiments predating commercially available computed tomography. Br J Radiol 1992;65:835-37

7. Araki K, Maki K, Seki K, et al. Characteristics of a newly developed dentomaxillofacial x-ray cone beam CT scanner (CB MercuRay): system configuration and physical properties. Dentomaxillofac Radiol 2004;33:51-59

8. Cohnen M, Kemper J, Mobes $\mathrm{O}$, et al. Radiation dose in dental radiology. Eur Radiol 2002;12:634-37

9. Mah JK, Danforth RA, Bumann A, et al. Radiation absorbed in maxillofacial imaging with a new dental computed tomography device. Oral Surg Oral Med Oral Pathol Oral Radiol Endod 2003;96:508-13

10. Mozzo P, Procacci C, Tacconi A, et al. A new volumetric CT machine for dental imaging based on the cone-beam technique: preliminary results. Eur Radiol 1998;8:1558-64

11. Sukovic P. Cone beam computed tomography in craniofacial imaging. Orthod Craniofac Res 2003;6 Suppl 1:31-36

12. Dalchow CV, Weber AL, Bien S, et al. Value of digital volume tomography in patients with conductive hearing loss. Eur Arch Otorhinolaryngo 2006;263:92-99

13. Dalchow CV, Weber AL, Yanagihara N, et al. Digital volume tomography: radiologic examinations of the temporal bone. AJR Am J Roentgenol 2006;186:416-23

14. Bartling SH, Gupta R, Torkos A, et al. Flat-panel volume computed tomography for cochlear implant electrode array examination in isolated temporal bone specimens. Otol Neurotol 2006;27:491-98

15. Majdani $\mathrm{O}$, Bartling $\mathrm{SH}$, Leinung $\mathrm{M}$, et al. A true minimally invasive approach for cochlear implantation: high accuracy in cranial base navigation through flat panel-based volume computed tomography. Otol Neurotol 2008;29:120-23

16. Nickoloff EL, Dutta AK, Lu ZF. Influence of phantom diameter, $\mathrm{kVp}$ and scan mode upon computed tomography dose index. Med Phys 2003;30:395-402

17. Rock C, Linsenmaier U, Brandl R, et al. Vorstellung eines neuen mobilen CBogen-/CT-Kombinationsgerät (ISO-C-3D). Erste Ergebnisse der 3D-Schnittbildgebung [Introduction of a new mobile C-arm/CT combination equipment (ISO-C-3D). Initial results of 3-D sectional imaging]. Unfallchirurg 2001;104:827-33

18. Rock C, Kotsianos D, Linsenmaier U, et al. Untersuchungen zur Bildqualität Hochkontrastauflösung und Dosis am Stamm- und Gliedmassenskelett mit einem neuen dedizierten CT-System (ISO-C-3D) [Studies on image quality, high contrast resolution and dose for the axial skeleton and limbs with a new, dedicated CT system (ISO-C-3 D)]. Rofo 2002;174:170-76 